\section{Carbon dioxide emissions}

SIR - Wigley and colleagues (Nature 379, 240 ; 1996) believe it would be preferable from an economic viewpoint to defer reducing carbon dioxide emissions for several decades rather than starting immediately. In comparing the economic costs of different reduction schedules, they invoke the cost-benefit criterion of the Framework Convention on Climate Change. They have, however, paid little attention to the 'benefits' aspect, because, they say, estimating the beneficial implications for agriculture, fisheries, biodiversity, human health and so on is a "highly uncertain" exercise.

This is certainly true. Many commentators argue, therefore, that conventional cost-benefit analysis in this complex context is inadequate, as it seeks to assign market valuations to entities that are not normally seen in financial terms, as well as to long-term effects that are often beyond the economist's usual discounting horizon.

Ethicists and political scientists may question both the propriety and political practicality of attempting to pass the cost of reducing emissions to future generations. More fundamentally, the complex, uncertain and potentially unfamiliar consequences of global climate change will require different, more flexible approaches to the assessment of risks and to social decision-making.

Wigley et al. ask whether a schedulerelated increase of around $0.2{ }^{\circ} \mathrm{C}$ in the global mean temperature over, say, a century would translate into "significantly higher damages", and, if so, whether this would offset the economic gains from delay. Most of the Intergovernmental Panel on Climate Change (IPCC) scientists who have struggled, in Working Group II, to identify and quantify the likely impacts of climate change on natural and social systems recognize that such high-resolution arithmetic is impossible. It has been hard enough working at tenfold lower resolution, assessing the likely effects of an overall $2.0^{\circ} \mathrm{C}$ rise in global mean temperature over the next century.

In the health impacts chapter of the impending IPCC Second Assessment Report (of which I am the convening lead author), we have identified the major changes in population health that are likely to occur. The effects include deaths from heat waves, altered patterns of vectorborne infectious diseases, some increases in regional malnutrition, problems from water shortages and flooding, and the consequences of population displacement. By their nature, the magnitude and costs of such effects (many of them mediated by climatic disturbances of complex ecological systems) cannot be calculated precisely.
Indeed, thresholds, feedback processes and interactions between the various factors that place stress on the environment could cause surprises.

Attempting to reduce the complexities of climate change, its impact on the biosphere, and the implications for human experiences to market-based values, and then to pass the bill to future generations in the hope that they will appreciate our farsighted capital accumulation strategy, is not a solution. Cost-benefit analysis is inadequate, and unadorned cost-only analysis more so. The issues posed by global environmental changes will require us to revise our scientific risk assessment and related decision-making.

\section{A. J. McMichael}

Department of Epidemiology

and Population Sciences,

London School of Hygiene

and Tropical Medicine,

Keppel Street, London WC1E 7HT, UK

\section{Prescribing oral contraceptives}

SIR - Michael Rawlins ${ }^{1}$ draws attention to errors in your leading article ${ }^{2}$ on the latest UK 'pill scare'. But one error, which he does not mention, acquires fresh relevance with the publication of interim data from the study of cardiovascular disease in oral contraceptive (OC) users directed by Professor Walter Spitzer ${ }^{3}$.

It is not correct, as your leading article stated, that 'third-generation' $O C$ were introduced to reduce the risk of venous thrombosis; their oestrogen content is essentially unchanged compared to secondgeneration products, and it is this fact that makes the increased risk of venous disease so surprising. Introduction of third-generation OC, containing desogestrel or gestodene progestagens, was in response to concern about the risks of arterial diseases such as myocardial infarction and stroke. Increased risk of myocardial infarction in OC users was predicted by one of us (V.W.) in 1966 (ref. 4), on the basis of biochemical changes. This possibility was confirmed by a number of epidemiological studies during the 1970s culminating in the Royal College of General Practitioners' Oral Contraception Study, which linked the dose of oral progestagen (norethisterone or levonorgestrel) with arterial disease mortality. At the same time, the progestagen component of some formulations was linked with low serum HDL concentrations. Because high-density lipoprotein (HDL) had been identified as a factor that could protect against coronary heart disease, this provided a rationale for OC-induced arterial disease. Subsequent reformulations were promoted on the basis of their ability to avoid an HDL-lowering effect. In the latter part of the 1980s the third-generation pills appeared, which increased HDL levels.

Evidence continues to accumulate in favour of a beneficial effect of increased HDL level on arterial disease, and, with the publication of myocardial infarction data from the Transnational Study ${ }^{3}$, we can now begin to see whether OC-induced changes in HDL may indeed be important. Use of second-generation $\mathrm{OC}$ was associated with a significant odds ratio for myocardial infarction of 3.1 (95\% confidence interval 1.5-6.3) compared with women not taking OC. The corresponding figures for thirdgeneration products were $1.1(0.4-3.4)$. The odds ratio for third- versus second-generation products was $0.4(0.1-1.2)$. These data were published prematurely due to the changes in UK prescribing practice resulting from the precipitate action of the Committee on the Safety of Medicines. They nevertheless provide impressive preliminary support for an improvement in arterial disease risk in users of third-generation pills. It is essential to keep these issues in mind in the intelligent and well informed collaborative decision making ${ }^{5}$ that should lie at the heart of modern OC prescribing.

lan F. Godsland

David Crook

Victor Wynn

Wynn Division of Metabolic Medicine,

(National Heart and Lung Institute, Imperial College),

21 Wellington Road,

London NW8 9SQ, UK

1. Rawlins, M. D. Nature 379, 202 (1996).

2. Nature 377, 663 (1995).

3. Spitzer, W. Br. med. J. 312, 88-90 (1996)

4. Wynn, V., Doar, J. W. H. \& Mills, G. L. Lancet Ii, 720-723 (1966).

5. McPherson, K. Br. med. J. 312, $68-69$ (1996).

\section{Not so public}

SIR - "There is no 'burying' of ORI's findings", writes Alan Price of the Office of Research Integrity. "ORI makes its findings very public" (Nature 379, 11; 1996). That is not so. Most of the complaints brought to ORI are dismissed without formal investigation (ORI 1994 Annual Report dated April 1995), and these findings are entirely concealed from the public.

For only the few complaints formally investigated does ORI issue public reports, and only if a complaint is upheld does the report identify the people and institution involved.

\section{Charles W. McCutchen}

5213 Acacia Avenue,

Bethesda,

Maryland 20814, USA 